psoriatic arthritis (PsA) and ankylosing spondylitis (AS) in North East Romania from 2003 to 2018.

Methods: We performed a hospital-based retrospective cohort study in consecutive adult patients receiving their first biologic agent (TNF or nonTNF drugs) according to local recommendations in two academic centers. Patients were classified based on the initial TB/latent TB screening test: the tuberculin skin test (positive if $>5 \mathrm{~mm}$, TST group) or interferon gamma release assays (positive if $>0.35 \mathrm{IU} / \mathrm{mL}$ QuantiFERON-TB gold, QFT group); retesting was done regularly if negative initial.

Data about drug efficacy was recorded every 24-weeks based on standard scores (DAS28-ESR for RA, DAPSA for PsA, ASDAS-CRP for AS), while TB risks at the end of the study or prior to switching were determined as hazard ratio $(\mathrm{HR})$ with $95 \%$ confidence interval $(\mathrm{Cl})$ using cox regression.

Statistical analysis was performed in SPSS-19, $p<0.05$, on subgroups of patients depending on whether positive TST or QFT at baseline.

Results: 673 patients (360 RA, 116 PsA, 195 AS) were recruited; 55 of them (33 RA, 13 AS, 9 PsA) had latent TB at baseline and received chemoprophylaxis with isoniazid before starting biologics according to local policy. Fourteen active TB were identified, the majority of them occurred within one year of biologics (ranging 6 to 52 months), as follows: three pulmonary TB in AS (two in etanercept, one adalimumab), one case of pulmonary TB in PSA (infliximab) and ten cases of RA (six pulmonary TB in abatacept, two adalimumab, etanercept and two infliximab; one ganglionar infection with infliximab; one peritoneal under certolizumab; one pulmonary and pleural under infliximab biosimilar; one pulmonary and peritoneal TB under adalimumab). The rate of active TB was 5/321.32 patient-years for infliximab, 4/594.64 for adalimumab, 1/38.39 patient-years for certolizumab, 3/721.51 for etanercept and 1/21.12 patient-years for abatacept, respectively. We reported an increased risk of TB disease in anti-TNF monoclonal antibodies users vs. soluble receptor, with an incidence ratio of $2.66(p<0.05)$. Interestingly, no significant TB risk factors were demonstrated in our cohort; baseline latent TB, previous active TB, chronic glucocorticoid use, high disease activity at baseline, comorbidities, concurrent synthetic drugs were not associated with increased risk $(p>0.05)$. In addition, we described 18 new patients with positive QFT when TB retesting as per protocol, classified as latent TB and requiring chemoprophylaxis.

Conclusion: The risk of tuberculosis remains a reality in biologics, despite extensive screening and prevention methods. The risk is variable with different drugs, higher with TNF inhibitors and relatively low in newer biologics, emphasizing a risk-stratification when selecting biologics in such patients.

Disclosure of Interests: CODRINA ANCUTA Speakers bureau: Abbvie, Pfizer, Novartis, MSD, Roche, Biogen, UCB, Lilly, Cristina Pomirleanu Speakers bureau: Abbvie, Pfizer, UCB, Georgiana Strugariu Speakers bureau: Abbvie, Pfizer, UCB, Luiza Petrariu Speakers bureau: Abbvie, Pfizer, Novartis, Raluca Paiu: None declared, Eugen Ancuta: None declared, Codruta Bran Speakers bureau: Abbvie, Pfizer, Novartis, Lilly, Roche, Rodica Chirieac: None declared

DOI: 10.1136/annrheumdis-2019-eular.3657

\section{FRI0077 SHORT DURATION OF CONVENTIONAL SYNTHETIC DISEASE-MODIFYING ANTI-RHEUMATIC DRUGS (CSDMARDS) BEFORE AND AFTER BECOMING A CSDMARD INADEQUATE RESPONDER IN RHEUMATOID ARTHRITIS PATIENTS}

Robin K Dore ${ }^{1}$, Jenya Antonova ${ }^{2}$, Huang Huan ${ }^{3}$, Magdaliz Gorritz ${ }^{3}$, Mark C. Genovese ${ }^{4} .{ }^{1}$ private practice, Tustin, CA, United States of America; ${ }^{2}$ Gilead Sciences, Foster City, CA, United States of America; ${ }^{3} I Q V I A$, Plymouth Meeting, $P A$, United States of America; ${ }^{4}$ Stanford University, Division of Immunology and Rheumatology, Stanford, CA, United States of America

Background: ACR guidelines recommend starting rheumatoid arthritis (RA) treatment with conventional-synthetic (cs)DMARD monotherapy ${ }^{1}$. If disease activity remains moderate or high, the guidelines recommend adding or switching to another csDMARD, biologic (b)DMARD, or janus kinase inhibitors (JAKi) for established RA. In early ( $<6$ months) RA, the guidelines recommend tumor necrosis factor inhibitors TNFi over JAKi and TNFi+methotrexate (MTX) over JAKi+MTX.

Objectives: This study aimed to investigate treatment patterns and duration in csDMARD IR patients.

Methods: In fully-adjudicated commercial medical and pharmacy health insurance claims database with 40 million lives annually, adult RA patients ( $\geq 2$ RA diagnoses $\geq 30$ days apart) who started csDMARD (1/1/ 2012-3/31/2017) and then switched to or added another DMARD (index date, ID) were selected. Real world treatment patterns of patients with 21-year continuous enrollment before (baseline) and after ID (index treatment) were assessed.

Results: Among 25,104 csDMARD initiators, 10,091 (40\%) demonstrated $\mathrm{IR}$, and 7,816 met all the inclusion and exclusion criteria (median age 54 years, $26 \%$ male). The baseline csDMARD treatment lasted for a median of 4.3 months and mostly comprised of monotherapy (96\%). Upon treatment regimen change, $62 \%(n=4,869)$ initiated combination therapy $(32 \%$ csDMARD+csDMARD, $28 \%$ csDMARD+TNFi, $1.4 \%$ csDMARD+other bDMARD, $0.5 \%$ csDMARD+JAKi) and $38 \%$ initiated monotherapy $(27 \%$ on csDMARD, $10 \%$ TNFi, $0.7 \%$ other bDMARD, $0.5 \%$ JAKi). Post-switch, the median treatment duration was longer for combination therapy than monotherapy: 13.7 vs. 5.2 months $(p<0.001)$. Among next therapies, csDMARD showed the trend towards shortest durability and JAKi - toward longest durability. Among monotherapies the median treatment duration varied: 4.9 (csDMARD), 5.9 (TNFi), and 8.1 (JAKi) months ( $\mathrm{p}=0.2$, comparing all). The median duration of combination therapies lasted: 12.5 (csDMARD +CSDMARD), 14.9 (TNFi+csDMARD), and 17.2 (JAKi+csDMARD) months $(p=0.04$, comparing all).

\begin{tabular}{lcc}
\hline Characteristics & \multicolumn{2}{c}{$\begin{array}{c}\text { csDMARD IRs } \\
\text { (n=7,816) }\end{array}$} \\
\hline Index therapy allocation after csDMARD IR, (n,\%) & $\mathbf{N}$ & $\%$ \\
\hline cDMARD Monotherapy & 2,095 & 27 \\
TNFi Monotherapy & 757 & 10 \\
Other bDMARD Monotherapy & 55 & 0.7 \\
JAKi Monotherapy & 40 & 0.5 \\
cDMARD + cDMARD Combination & 2,539 & 32 \\
TNFi + cDMARD Combination & 2,178 & 28 \\
Other bDMARD + cDMARD Combination & 112 & 1.4 \\
JAKi + cDMARD Combination & 40 & 0.5 \\
Index treatment duration after csDMARD IR, months, median (IQR) & Months & $\mathbf{( I Q R )}$ \\
Monotherapy & 5.2 & 9.8 \\
csDMARD & 4.9 & 10.1 \\
TNFi & 5.9 & 10.1 \\
JAKi & 8.1 & 11.9 \\
Combination therapy & 13.7 & 15.6 \\
csDMARD+csDMARD & 12.5 & 14.8 \\
TNFi+csDMARD & 14.9 & 15.9 \\
JAKi+csDMARD & 17.2 & 11.3 \\
\hline
\end{tabular}

Conclusion: The real-world evidence suggests that treatment durability may be better for JAKi than TNFi (both monotherapy and combination). The majority of cSDMARD patients switched to another csDMARD, which showed short durability of treatment, suggesting that switching MOA may benefit these patients.

\section{REFERENCE:}

[1] Singh JA, et al. 2015 American College of Rheumatology Guideline for the Treatment of Rheumatoid Arthritis. Arthritis Rheumatol. 2016 Jan;68(1):126

Disclosure of Interests: Robin K Dore Grant/research support from: Gilead Sciences, AbbVie, Amgen, Lilly, Pfizer, Regeneron, Sanofi, Consultant for: AbbVie, Amgen, Lilly, Speakers bureau: AbbVie, Amgen, Lilly, Sanofi, Regeneron, Pfizer, UCB, Jenya Antonova Shareholder of: Gilead Sciences, Employee of: Eli Lilly and Company, Medimmune, Genentech, Gilead Sciences, Huang Huan Grant/research support from: Gilead Sciences, Magdaliz Gorritz Grant/research support from: Gilead Sciences, Mark C. Genovese Grant/research support from: Sanofi/Genzyme, Genentech/ Roche, RPharm, Consultant for: Sanofi/Genzyme, Genentech/Roche, RPharm

DOI: 10.1136/annrheumdis-2019-eular.1848

\section{FRI0078 \\ IMPACT OF THE IMPLEMENTATION OF FLUSH SERUM ON TROUGH LEVELS OF INFLIXIMAB IN PATIENTS WITH INFLAMMATORY JOINT DISEASE}

Marta Bassas Palou, Cristina Gonzalez, Ana Atares, Raimón Sanmartí, Julio Ramirez. Hospital Clinic Barcelona, Rheumatology, Barcelona, Spain

Background: The administration of intravenous biological therapies is based on the manufacturer's instructions and the administration guidelines of Spanish Medicine Agency. We observed that a considerable amount of the solution remains in the tubing of the equipment after the administration of the drug. This amount depends on the drug and the solution used for the infusion. In the particular case of Infliximab is around $7 \%$ of the total solution to infuse, so it could potentially have a relevant therapeutic impact. 